\title{
Comparison of ANN and SVM to Identify Children Handwriting Difficulties
}

\author{
Anith Adibah Hasseim, Rubita Sudirman, Puspa Inayat Khalid, Narges Tabatabaey-Mashadi \\ Faculty of Electrical Engineering, Universiti Teknologi Malaysia, Johor Bahru, Johor, Malaysia \\ Email: rubita@fke.utm.my
}

Received 2013

\begin{abstract}
This paper compares two classification methods to determine pupils who have difficulties in writing. Classification experiments are made with neural network and support vector machine method separately. The samples are divided into two groups of writers, below average printers (test group) and above average printers (control group) are applied. The aim of this paper is to demonstrate that neural network and support vector machine can be successfully used in classifying pupils with or without handwriting difficulties. Our results showed that support vector machine classifier yield slightly better percentage than neural network classifier and it has a much stable result.
\end{abstract}

Keywords: Neural Network; Support Vector Machine; Handwriting Difficulties

\section{Introduction}

Handwriting is the primary form of written expression for young elementary school students. Handwriting has long been an effective means to record information, transmit message and project feelings [1] for communication among people. There is evidence to indicate that at least $10 \%-30 \%$ of children who have difficulty with handwriting and need to be resolved with the right intervention [2]. Most of the studies that involve in handwriting movement only focused on children with known psychological or physical problem. Yet, not all problems can be categorized as clear cut disease and condition [3]. Various softwares have been presented for handwriting recognition and movement analysis, but softwares directly related to child handwriting analysis with the prospective of screening children in general, and addressing difficulties are rare and the research is in its early stage.

The development of children's writing ability is important in building self-esteem among children. For example, in 1998 Graham and Weintraub reported that students with poor handwriting needed twice as much time to copy a written passage as those with good handwriting [4]. Hence, difficulty in writing for young student can lead to a dislike of writing, frustration with writing, and development of a negative mind-set about writing ability. As a result, this will truly limit their further writing development and subsequently retarded to academic success. Therefore, early analysis of those children will benefit the educational system in order to provide an instructional handwriting program suited to their strengths and weaknesses. It is evident that educators and mental health experts are in need of empirically-based assessment and intervention procedures to help identify and treat children with writing disorders.

\subsection{Evaluation of Handwriting Difficulties}

Several studies have been done to evaluate early writing skill in primary grade. These research findings were investigated and describes in term of legibility and speed, see $[4,5]$. However, the scarcity of valid and reliable handwriting evaluation tools, the complexity in the scoring tools and the long processing time by the evaluator who needs to judge the writing product for each of the legibility criteria, limit the application of standardized assessments in the evaluation of handwriting difficulties in clinical and classroom setting [2]. In addition, in most of the mild cases, the symptoms of handwriting difficulty are present but normally are not recognised by the teachers or certified evaluators.

In this paper we describe experiments carried out using two classification methods in classifying children with and without handwriting problem based on drawing tasks. In contrast with similar method known by Khalid in [6] and other related studies $[7,8]$ we tested each different feature individually and describe experiments carried out using Support Vector Machine (SVM) in addition to those classification methods used in previous researches. SVM is a supervised learning method that has proven it's efficiently over classic Neural Networks and its subset [9]. The advantages of SVM are good gener- 
alization performance, able to handle high dimensional data and able to map the data into new high dimensional feature space for better classification using kernel functions. The aim of this paper is to present that SVM and ANN can be effectively used as an automated system in seeking out pupils with handwriting problem.

\subsection{Artificial Neural Network (ANN)}

Probably neural network methods are most widely known. An ANN can be define as information processing concept that is inspired by the way biological nervous systems, such as the brain, process information. This system can be seen in an architecture inspired by the structure of the cerebral cortex of the brain [10]. These processing elements are usually organized into a sequence of layers with entire or random connections between the layers.

Basically, ANN is divided to three layers which are an input layer, at least one hidden layer and an output layer. Multilayer feedforward network is the simplest of ANN devise. It can be used to model some mapping between sets of input and output variable with appropriate pattern of weights. Figure 1 shows a basic diagram of feedforward neural network which can be trained using back propagation method, supervised learning network.

Back propagation learning uses the gradient descent procedure to modify the connection weights which is derived from the consideration of minimizing some error function. This error function is needed to change the network parameter, which is advantage in improving the network performance.

\subsection{Support Vector Machine(SVM)}

We now describe the basic idea of Support Vector Machine, more explanation can be found in $[9,11,12]$. SVMs are new technique suitable for binary classification tasks. In addition, SVM is one of the excellent tools for classification and regression problems with a good generalization performance.

SVM constructs a hyperplain or a set of hyperplains to separate the two sets of data in a feature space. The key approach of SVM is to try finding the best hyperplain by maximizing the minimum margin between the two sets. An optimal separating hyperplane is shown in Figure 2. In SVM, training vectors are mapped into higher dimensional space by the function of $\varphi$ which given a training set of instance-label pairs $\left(x_{i}, y_{i}\right) ; i=1, \ldots, l$ where $x_{i} \in \mathrm{R}^{\mathrm{n}}$ and $y \in\{1,-1\}^{l}$. All operations in learning and testing modes are done using an appropriate kernel functions which is define as $K\left(x_{\mathrm{i}}, x\right)=\varphi^{\mathrm{T}}\left(x_{\mathrm{i}}\right) \varphi(x)$ [12]. For example, polynomial kernel with 2 orders $K(x, x)=\left(x, x^{T}+1\right)^{2}$ map the 2-dimensional space $\left\{\left(x_{1}, x_{2}\right) \mid x_{1}, x_{2} \in R\right\}$ into 6-dimensional space $\left\{\left(x_{1}, x_{2}, \sqrt{2} x_{1} x_{2}, \sqrt{2} x_{1}, \sqrt{2} x_{2}, 1\right) \mid\right.$ $\left.x_{1}, x_{2} \in R\right\}$ Furthermore, kernel function has an important effect on the functional efficiency of SVM. The popular kernel functions include Gaussian radial basis function, polynomial and sigmoidal functions.

\section{Method}

\subsection{Datasets}

Our sample target populations are general students who are beginning to write. The data for this research was obtained from Khalid et al in [13]. This sample consisted of 120 first grade children who assigned to two groups of writers, below average printers (test group) and above average printers (control group). There were 60 pupils in the control group and 60 pupils in the test group. Each participants were required to complete 8 drawing task; vertical downward (VD), vertical upward (VU), horizontal rightward (HR), horizontal leftward (HL), right oblique downward (RD), right oblique upward (RU), left oblique downward (LD), and left oblique upward (LU) as shown in Figure 3. These drawings are the most basic drawing and the most common and effective means of communication that have been applied in various application for more than a decade. In a simple sense, these line drawings are a picture that convey to their viewer information through the shape, size and manner

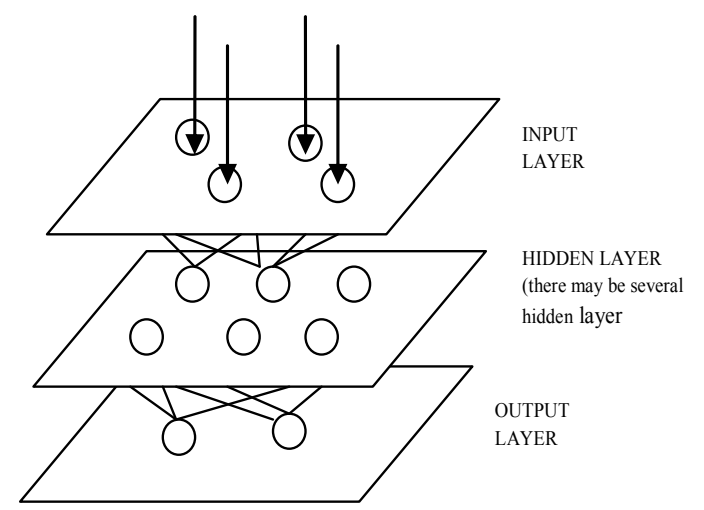

Figure 1. A simple neural network diagram.

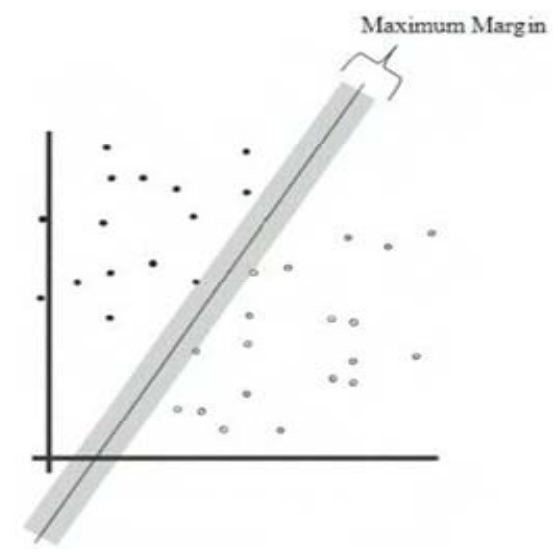

Figure 2. Optimal separating hyperplain. 


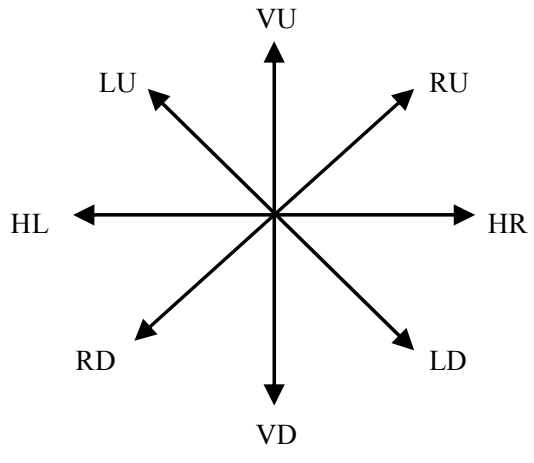

Figure 3. A notion of eight directions.

of interconnection of thin lines on a contrasting background [14].

Dynamic data (such as velocity and pressure values) of drawing performance have been rigorously studied in recent researches e.g., $[15,16]$. Those studies have shown that dynamic data also affect the performance characteristic of drawing tasks besides the used of static data. For our experiments, 2 features were selected to be used in the classification process:

1) Feature 1: The standard deviation of pen pressure when drawing $\mathrm{RU}, \mathrm{p}$-value $<0.0001$ and $\mathrm{z}$-value $=$ minus 4.319 and,

2) Feature 2: Ratio of time taken to draw $\mathrm{HR}$ and HL, $\mathrm{p}$-value $<0.0001$ and $\mathrm{z}$-value $=$ minus 5.205.

\subsection{Architecture}

\subsubsection{Artificial Neural Network Classification}

Artificial Neural Network training was developed using MATLAB 7.6 software. The network chosen for the prediction neural network had one input layer, 2 hidden layers and one output. For the hidden layers, the number of neurons is obtained by trial and error. The most compact network is chosen and presented. The network training parameters are:

- Training algorithm : Gradient descent with momentum training

- Perform function : Mean Square Error

- Training goal achieved : 0.04

- Training epochs : 10000

- Training momentum constant : 0.95

- Learning rate: 0.2

- Ratio to increase learning rate: 1.05

- Ratio to decrease learning rate: 0.7

The hidden layers and the output layer used log sigmoid activation function which it calculate a layer's output from its net input. This function generates outputs between 0 and 1 as the neuron's net input could be any values from negative to positive infinity. The threshold, $\theta$ for the output was set to 0.5 . Therefore if the final testing value exceeds the threshold value, the function will takes the value 1 and 0 otherwise. The flow chart of training network using BP algorithm is shown in Figure 4.

Since this dataset is large, we used cross validation method to test our classifiers. The data is randomly portioned into 10 equally size folds. In each folds, we selected 6 samples from the control group and 6 samples from the test group. Next, one fold is used for testing while the remaining 9 folds are used for validation. This process is continued 10 times such that within each itertion a different fold of the data is held-out for validation and the rest folds are used for learning.

As the initial weight of the each training process is not fixed, the network could give slightly different results. Hence, we trained each algorithm with 10 trials and get the average performance of the model.

\subsubsection{Support Vector Machine Classification}

The SVM was also run by using program MATLAB 7.6. Total 120 samples are used as input signals. Linear SVM is used as kernel function for training SVM. Usually among popular kernels, the linear kernel is much faster in training and testing speed. An important advantage of linear classification is that training and testing procedures are much more efficient. Therefore, linear classification can be very useful for some large-scale applications [17].

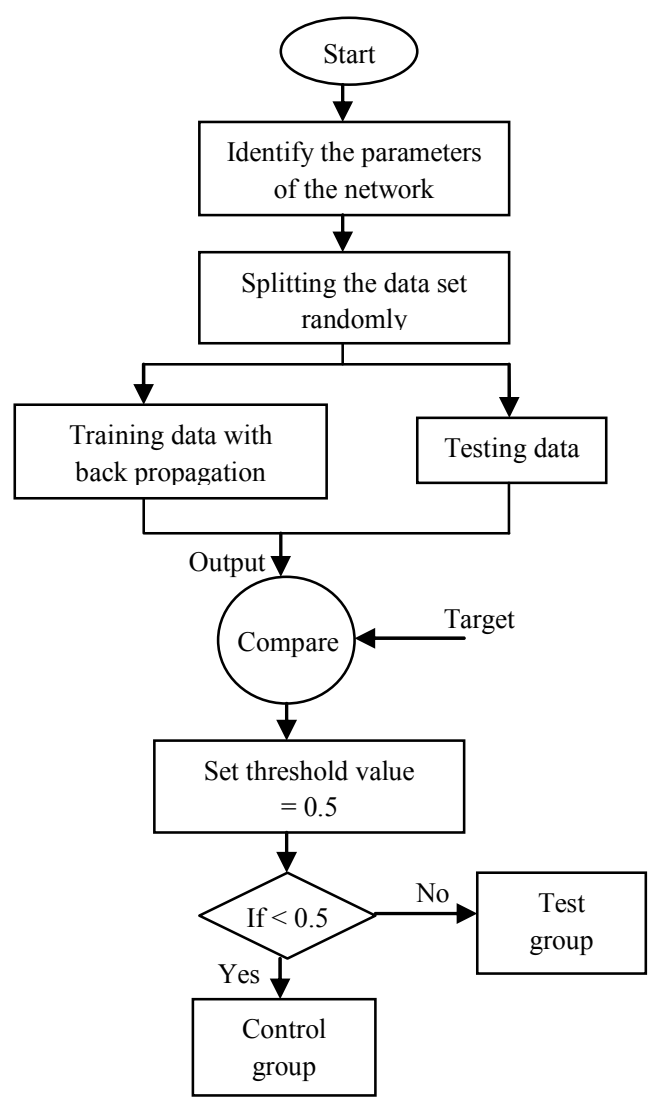

Figure 4. Flowchart for identification of the handwriting problem using artificial neural network model. 
The 12 random signals are selected from the data used to test SVM and the remaining data used for training. Just like neural network, the experiment was done with 10 iterations of 10-fold cross validation and the final average performance of the classifier was taken out.

\section{Results and Discussions}

Table 1 shows the classification results obtained for each attributes using ANN classifier and SVM classifier. The classification performance was divided into 2 parts: control and test. Control performance was based on the classification rate of the samples from the control group in the testing set while test performance was based on the classification rate of the samples from the test group in the testing set. The classification was considered correct if the output from the model was similar to the one that had been judged by the teachers (using Handwriting Proficiency Screening Questionnaire (HPSQ)).

In this paper, we used the classification error (rejection of genuine category) as the metric. From Table 1, we can see that the classification rate for SVM is better than ANN in both cases; control group and test group with the average accuracy of classificatory testing based on SVM algorithm reaches more than $83 \%$.

Based on the observation, neural network not only provide weakly performance in regression problem but also require more computational time than SVM technique. The recognition rates for SVM also show a significant improvement compared to ANN. Moreover the classificatory result of the SVM algorithm is more stable since it is not easily influenced by primal weighting value like neural network. In addition, the advantage of this approach clearly lies in its simplicity since no parameter has to be tuned. Overall, SVM is more convenient and superior when it comes to identify and assess "poor" writers.

Furthermore, between the two features, the results indicated that feature 1 which is the standard deviation of pen pressure when drawing RU is better than feature 2 (ratio of time taken to draw HR and HL) if we measure the percentage of correctly classified performance of control group. However, if the measure of performance was percentage of test group correctly classified, the feature 2 outperformed the feature 1 .

Table 1. Classification result.

\begin{tabular}{cccc}
\hline \multicolumn{2}{c}{ Classifiers } & Feature 1 & Feature 2 \\
\hline \multirow{2}{*}{ ANN Classifier } & Control group (\%) & 86.67 & 75.00 \\
& Test group (\%) & 58.88 & 63.33 \\
\multirow{2}{*}{ SVM classifier } & Control group (\%) & 91.67 & 83.33 \\
& Test group (\%) & 83.33 & 83.33 \\
\hline
\end{tabular}

\section{Conclusions}

An experiment study of classification performance with the aim of identifying children with and without handwriting difficulties has been presented. It is based on the handwriting proficiency screening questionnaire (HPSQ). However, the collected data from the questionnaire is normally subjective and imprecise. Thus, the experiment can be further improve by using dynamic data that is both sensitive and specific is suggested for the screening process to be effective.

Two techniques; ANN and SVM have been used in this study to select those who are at risk of handwriting difficulty due to the improper use of graphic rules. Here, it can be concluded that both methods are able to classify students with or without handwriting difficulties. However, the performances of the two classifiers are different, SVM technique is more effective and doable way than ANN method which it gives the average classification rate more than $83 \%$.

\section{Acknowledgements}

This work was supported by the Malaysia Ministry of Higher Education and Universiti Teknologi Malaysia under Vote Q.J130000.2623.09J28.

\section{REFERENCES}

[1] S. Chu, "Occupational Therapy for Children with Handwriting Difficulties: A Framework for Evaluation and Treatment," British Journal of Occupational Therapy, Vol. 60, 1997, pp. 514-520.

[2] K. P. Feder and A. Majnemer, "Handwriting Development, Competency, and Intervention," Developmental Medicine Child Neurology, Vol. 49, No. 4, 2007, pp. 312-317. doi.org/10.1111/j.1469-8749.2007.00312.x

[3] R. Sudirman, N. T. Mashadi and I. Ariffin, "Aspects of A Standardized Automated System for Screening Children's Handwriting," First International Conference on Informatics and Computational Intelligence, Bandung, 12-14 December 2011, pp. 49-54. doi:10.1109/ICI.2011.19

[4] S. Graham, V. W. Berninger, N. Weintraub and W. Schafer, "Development of Handwriting Speed and Legibility in Grades 1-9," Journal of Educational Research, Vol. 92, No. 1, 1998, pp. 42-52. doi:10.1080/00220679809597574

[5] S. M. Koziatek and N. J. Powell, "Pencil Grips, Legibility, and Speed of Fourth-Graders' Writing in Cursive," American Journal of Occupational Therapy, Vol. 57, No. 3, 2003, pp. 284-288. doi:10.5014/ajot.57.3.284

[6] P. I. Khalid, "Handwriting Ability Assessment Model Using Dynamic Characteristics of Drawing Process," PhD Thesis, Universiti Teknologi Malaysia, 2012.

[7] R. M. Guest, S. Chindaro, M. C. Fairhurst and J. M. Potter, "Automatic Classification of Hand Drawn Geometric Shapes Using Constructional Sequence Analysis," Sev- 
enth International Conference on Document Analysis and Recognition (ICDAR'03) icdar, Vol. 2, 2003, p. 990.

[8] S. Chindaro, R. M. Guest, M. C. Fairhurst and J. M. Potter, "Assessing Visuo-Spatial Neglect Through Feature Selection and Combination from Geometric Shape Drawing Performance and Sequence Analysis," International Journal of Pattern Recognition \& Artificial Intelligence, Vol. 18, 2004, pp. 1253-1266. doi:10.1142/S0218001404003757

[9] C. J. C. Burges, "A Tutorial on Support Vector Machines for Pattern Recognition," Data Mining and Knowledge Discovery, Vol. 2, 1998, pp. 121-167. doi:10.1023/A:1009715923555

[10] L. H. Tsoukalas and R. E. Uhrig, "Fuzzy and Neural Approaches in Engineering," John Wiley and Sons, 1997.

[11] V. N. Vapnik, "The Nature of Statistical Learning Theory," Springer, New York, 1995. doi:10.1007/978-1-4757-2440-0

[12] C. W. Hsu, C. C. Chang and C. J. Lin, "A Practical Guide to Support Vector Classification," Technical Report, Department of Computer Science and Information Engineering, National Taiwan University, 2003.
[13] P. I. Khalid, J. Yunus, R. Adnan, M. Harun, R. Sudirman and N. H. Mahmood, "The Use of Graphic Rules in Grade One to Help Identify Children At Risk of Handwriting Difficulties," Research in Developmental Disabilities, Vol. 31, 2010, pp. 1685-1693. doi:10.1016/j.ridd.2010.04.005

[14] H. Freeman, "Computer Processing of Line-Drawing Images," ACM Computing Surveys, Vol. 6, 1974, pp. 57-97. doi:10.1145/356625.356627

[15] R. M. Guest, M. C. Fairhust and J. M. Potter, "Diagnosis of Visuo-Spatial Neglect Using Dynamic Sequence Features from A Cancellation Task," Pattern Recognition Analysis \& Applications, Vol. 5, 2012, pp. 261-270. doi:10.1007/s100440200023

[16] P. I. Khalid, J. Yunus and R. Adnan, "Extraction of Dynamic Features from Hand Drawn Data for the Identification of Children with Handwriting Difficulty," Research in Developmental Disabilities, Vol. 31, 2010, pp. 256-262. doi:10.1016/j.ridd.2009.09.009

[17] G. X. Yuan, C. H. Ho and C. J. Lin, "Recent Advances of Large-Scale Linear Classification," Proceedings of the IEEE, Vol. 100, No. 9, 2012, pp. 2584-2603. doi:10.1109/JPROC.2012.2188013 